\title{
Graphene-Supported Microwell Liquid Cell for In Situ Electron Microscopy in Materials Science
}

\author{
Andreas Hutzler ${ }^{1}$, Robert Branscheid ${ }^{2}$, Michael P.M. Jank ${ }^{3}$, Lothar Frey ${ }^{1,3}$ and Erdmann Spiecker ${ }^{2}$ \\ 1. Chair of Electron Devices (LEB), Friedrich-Alexander University Erlangen Nuremberg, Erlangen, \\ Germany. \\ 2. Institute for Micro- and Nanostructure Research (IMN) and Center for Nanoanalysis and Electron \\ Microscopy (CENEM), Friedrich-Alexander University Erlangen Nuremberg, Erlangen, Germany. \\ 3. Fraunhofer Institute for Integrated Systems and Device Technology Erlangen, Germany.
}

Liquid cell transmission electron microscopy (LCTEM) is a wide-ranging characterization method for dynamic processes in fluid environments. It is applied in a variety of scientific fields like materials science, physics as well as electrochemistry and biology because it facilitates in situ imaging with a high temporal and spatial resolution [1]. To date there exist three main concepts of LCTEM and several derivatives of them: The commercially available, cost intensive liquid flow systems, the silicon-based static liquid cell and the graphene liquid cell. An advantage of the two latter ones is that, depending on the layout, they can be applied with conventional TEM specimen-holders. In case of the silicon-based static liquid cell, the achievable resolution is limited due to the membranes consisting of considerably thick silicon nitride and the channel depth defining the thickness of the liquid specimen that has to be penetrated by the electron beam. In addition to that, the observable area is a limiting factor, because the lateral TEM-window dimensions has to be traded off against window bulging, which is also depending on the membrane thickness. The ability of applying analytical techniques like EDX and EELS is very restricted as well, based on the fabrication steps that have to be used. These disadvantages of the known bulk micromachined cells can be circumvented using the graphene liquid cell design shown by Yuk et al. [2], where the silicon nitride membranes are replaced by the ultra-thin, electrically conductive low-Z material graphene. In the first instance, this reduces charging effects and the achievable resolution is perceivably enhanced. Furthermore, the observable area is much larger due to the utilization of conventional TEM-grids as carrier frame. However, one major drawback is that the liquid film thickness cannot be controlled and is a matter of coincidence. Moreover, the attainable success rate of specimen preparation is also lower than in the case of silicon-based static liquid cells.

In our approach, we make use of a combination of the liquid cell design used by Dukes et al. [3] and the graphene liquid cell [2] which is shown in figure 1. This design benefits from an increased observable area, which is defined by apertures etched into the mechanically stable silicon frame by anisotropic patterning of silicon. Difficulties with eucentric alignments are also largely suppressed because silicon edges only appear on one side of the frame. The liquid cell is also compatible with narrow pole piece gaps (e.g. super-twin lens in FEI microscopes) without the need of a specialized sample-holder due to the reduced total thickness in contrast to bonded designs. Besides that it is possible to apply a very thin silicon nitride membrane (i.e. $20 \mathrm{~nm}$ ) as a result of window bulging being minimized by decreasing the well-diameter. The well-depth defines the thickness of the liquid specimen and the multilayer graphenemembrane on the upper side can reduce radiation damage and improves the achievable spatial resolution to a maximum value. In detail, a resolution of $1.0 \AA$ could be demonstrated by using the liquid cell in a FEI $\operatorname{Titan}^{3}$ Themis operated at $300 \mathrm{kV}$ accelerating voltage (cf. figure 2). Spectroscopic methods like EDX are also highly improved in contrast to other static liquid cell designs because there is no shadowing from the cell-frame itself due to the flat front side. We could also demonstrate the ability of 
utilizing this liquid cell design inside an SEM. An experiment was conducted using a FEI Helios Nanolab 660 which was operated in transmission mode at a primary electron energy of $20 \mathrm{keV}$. Dendrite growth from $\mathrm{HAuCl}_{4}$-solution could be observed which was highly gained in contrast to TEMexperiments induced by the interaction volume being located inside the liquid specimen in most instances. Altogether this makes the liquid cell design versatile for various applications that also include not only material scientific, but also biological, medical or electrochemical matters by disposing minor changes in cell-geometry or attaching electrical or thermal contacting [4].

\section{References:}

[1] H-G Liao and H Zheng, Annual Review of Physical Chemistry 67 (2016), p. 719.

[2] J M Yuk et al, Science 336 (2012), p. 61.

[3] M J Dukes et al, Microscopy and Microanalysis 20 (2014), p. 338.

[4] Financial support by the DFG via the Research Training Group GRK1896 "In situ microscopy with electrons, X-rays and scanning probes" is gratefully acknowledged.

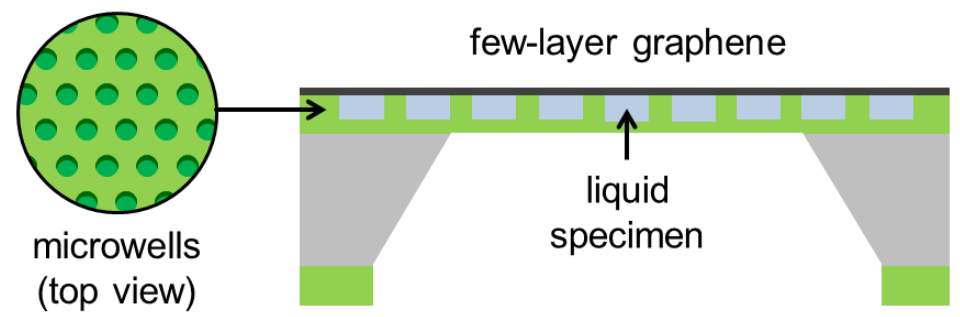

microwell containing membrane

silicon frame

mask-layer for micromachining

Figure 1. Schematic representation of a graphene-supported microwell liquid cell used for in situ electron microscopy of liquid specimen.
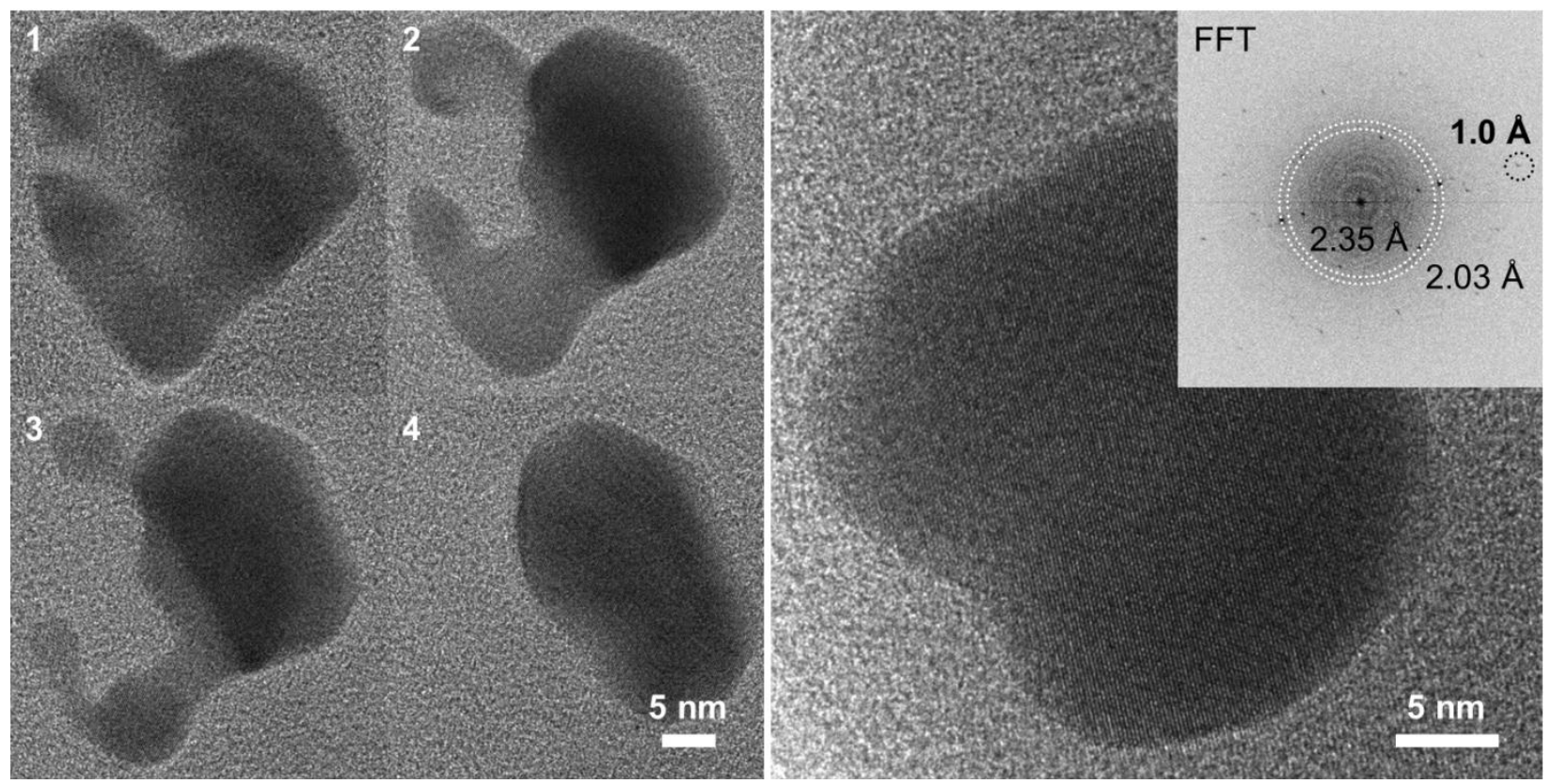

Figure 2. Image sequence of in situ liquid cell HRTEM showing a dissolving Au-nanoparticle in 10 $\mathrm{mM} \mathrm{HAuCl}_{4}$-solution and demonstration of the achievable resolution in the liquid cell (well depth: 180 nm, well diameter: $5 \mu \mathrm{m})$. 